\title{
Comportamiento óptico no-lineal de nanocristales semiconductores de CdS en una matriz de gel de sílice
}

\author{
N. DE LA ROSA-FOX, M. PIÑERO' ${ }^{1}$, R. LITRÁN Y L. ESQUIVIAS \\ Dpto. Física Materia Condensada. Facultad de Ciencias. Universidad de Cádiz. \\ ${ }^{1}$ Dpto. Física Aplicada. CASEM. Universidad de Cádiz. \\ 11510 Puerto Real (Cádiz)
}

\begin{abstract}
La inclusión de nanocristales en una matriz dieléctrica puede realizarse mediante el método sol-gel, permitiendo el control de parámetros cinéticos en el crecimiento de los cristales. El tamaño y distribución de dichos nanocristales se han estudiado por medio de difusión de neutrones a bajo ángulo (SANS), con una resolución espacial entre 1 y 50 nm. Bajo excitación óptica de alta intensidad, usando un láser (Nd:YAG) con pulsos de 6 ns y una frecuencia de repetición de $10 \mathrm{~Hz}$, se focalizó en la muestra para registrar la señal de fotoluminiscencia en la dirección perpendicular. El espectro indica procesos radiativos debidos a transiciones intrínsecas $(2.76 \mathrm{eV})$ y una banda ancha a más baja energía $(1.65 \mathrm{eV})$ correspondiente a recombinaciones en los defectos superficiales. La señal de fotoluminiscencia crece superlinealmente dando lugar a una amplificación (60 $\mathrm{cm}^{-1}$ ) debida principalmente a biexcitones que indican un comportamiento óptico no-lineal de los nanocristales de CdS.
\end{abstract}

Palabras clave: sol-gel. semiconductor CdS, fotoluminiscencia, SANS, ganancia óptica.

Non-linear optical behaviour of CdS semiconductor nanocrystals inside a silica gel matrix.

Nanocrystals embedded in a dielectric matrix can be obtained through sol-gel method, it permits the control of several kinetic parameters during the nanocrystal growth. The size and size distribution have been studied by means small-angle neutron scattering techniques (SANS), that allow a spatial resolution from 1 to $50 \mathrm{~nm}$. High intensity optical excitation from a Nd:YAG laser ( $6 \mathrm{~ns}$ pulse at $10 \mathrm{~Hz}$ repetition rate) was focused on the sample to study the photoluminescence yield at rigth angle. The spectrum shows radiative process from intrinsic transitions $(2.76 \mathrm{eV})$ and a broad band in the lower energy side $(1.65 \mathrm{eV})$ that correspond to crystal surface traps. The photoluminescence yield grows superlineary giving rise to amplified intensity $\left(60 \mathrm{~cm}^{-1}\right)$ due to biexciton species, this fact can be interpreted as a non-linear optical behaviour coming from the CdS nanocrystals.

Keywords: sol-gel, CdS semiconductor, photoluminescence, SANS, optical gain.

\section{INTRODUCCIÓN.}

El confinamiento cuántico de portadores en nanocristales semiconductores (quantum dots) se produce cuando el tamaño de los mismos es del orden, incluso menor, que el radio del de Bohr exciton, $32 \AA$ para el CdS $(1,2,3)$. Bajo fotoexcitación se crean parejas electrón-hueco, ya sean 1 como en el caso de los excitones, 1EHP, o bien 2 como los biexcitones, 2EHP, estos portadores cambian radicalmente el comportamiento óptico del material, dando lugar a efectos ópticos no-lineales, incrementándose cuando estos nanocristales están dispersos en una matriz dieléctrica, gel o vidrio de $\mathrm{SiO}_{2^{\prime}}(4,5)$. Estos efectos se ponen de manifiesto en determinadas condiciones experimentales debido al apantallamiento del potencial de Coulomb, así la obtención de un espectro de variación de la absorción por medio de técnicas de bombeo-sonda indica un índice de refracción no-lineal (6). También es indicativo de dicho comportamiento la existencia de ganancia óptica neta en el material debida al llenado de las bandas y creación de biexcitones (7). La distorsión del rayo como consecuencia de una ionización de los portadores es causa también de la aparición de efectos ópticos no-lineales (8).
La fabricación de estos materiales por la vía sol-gel mejora algunos parámetros como son: la densidad y homogeneidad de los cristales, su tamaño en el rango nanométrico, transparencia óptica de la matriz y superficies pasivas de los cristales $(9,10,11)$. Por otro lado, el método sol-gel permite adicionalmente el control del crecimiento del cristal a partir de su precipitación por sobresaturación en el estado líquido, sol, que posteriormente polimeriza según un entramado poroso como matriz de sílice en el que se encuentran encapsulados los cristales.

\section{PROCEDIMIENTO EXPERIMENTAL}

Durante el procesado hemos utilizado algunos aditivos químicos que controlan el proceso de envejecimiento del gel, tales como la Formamida. El método sol-gel implica una mezcla de los reactivos TMOS: $\mathrm{H}_{2} \mathrm{O}(\mathrm{pH}=1)$ :Formamida en una relación molar 1:4:7. Como fuente de los iones $\mathrm{Cd}^{+2}$ se utilizó acetato de cadmio (12). La gelificación tiene lugar a temperatura ambiente en recipientes herméticamente cerrados dejándose envejecer una semana. La evaporación del líquido de sinéresis se realiza en estufa a $50^{\circ} \mathrm{C}$ en atmósfera seca. Los 
geles monolíticos que se obtuvieron se trataron térmicamente en aire a temperaturas entre $200-500^{\circ} \mathrm{C}$, una vez enfriadas las muestras fueron expuestas a una corriente de $\mathrm{H}_{2} \mathrm{~S}$ a $150^{\circ} \mathrm{C}$ durante $1 \mathrm{~h}$, transformándose así el CdO en CdS. Las muestras presentaron buena resistencia mecánica así como buena transparencia óptica. En lo sucesivo seguiremos la nomenclatura matriz $+\mathrm{CdO}$ y matriz $+\mathrm{CdS}$, respectivamente.

En este estudio presentamos medidas de dispersión de neutrones a bajo ángulo (SANS), para el cálculo del tamaño y la distribución de los cristales en el interior del gel (13). Las curvas de intensidad de SANS en función del vector de difusión q fueron medidas desde 0.03 hasta $3 \mathrm{~nm}^{-1}$ para varias distancias muestra detector y una longitud de onda de los neutrones de $0.6 \mathrm{~nm}$. Utilizando una apertura de $7 \mathrm{~mm}$ de diámetro, la transmisión de ambas muestras fue superior al $80 \%$. Finalmente, la sección transversal macroscópica de la difusión coherente en $\mathrm{cm}^{-1}$ se obtiene utilizando la difusión del $\mathrm{H}_{2} \mathrm{O}$. Las medidas fueron realizadas en la estación de trabajo V4 del Berlin Scattering Neutron Center ubicado en el Hahn-Meitner Institut (Alemania).

Por otro lado, con el fin de comprobar el comportamiento óptico no-lineal de estos materiales se midió la señal de fotoluminiscencia bajo excitación óptica intensa. Para la excitación óptica se utilizó la línea de $355 \mathrm{~nm}$ de longitud de onda procedente de un láser pulsado de Nd:YAG, pulsos de $6 \mathrm{~ns}$ con una frecuencia de repetición de $10 \mathrm{~Hz}$. Para las medidas se utilizó la técnica de rendija de longitud variable, VSL, (14), que consiste en enfocar sobre la muestra el haz puntual del láser a través de una lente cilíndrica, mediante la variación de la longitud de la rendija formada en la superficie de la muestra se registra el espectro de fotoluminiscencia en la dirección perpendicular. La señal se focaliza sobre la rendija de entrada, $100 \mu \mathrm{m}$, de un analizador óptico multicanal, OMA, equipado a la salida con un detector lineal de fotodiodos, cuya superficie se mantiene a $5^{\circ} \mathrm{C}$ y se la hace pasar un flujo de $\mathrm{N}_{2}$.

\section{RESULTADOS Y DISCUSIÓN.}

La Fig. 1 muestra las intensidades de la difusión de neutrones para las muestras estudiadas, matriz $+\mathrm{CdO}$ y matriz $+\mathrm{CdS}$, respectivamente. La diferencia en intensidad indica una mayor difusión de la matriz+CdO debido a un efecto combinado de mayor número de centros difusores, porosidad, y sobre todo un mayor contraste de la densidad de longitud de difusión $\left(\Delta \rho_{\mathrm{b}}\right)^{2}$ del CdO. Sin embargo, dada la similitud de la forma entre ambas curvas parecen indicar que poseen tamaños similares, notándose en ambas una región de Guinier bastante amplia, $0.03-0.5 \mathrm{~nm}^{-1}$, lo que indica una gran homogeneidad de tamaños. En este sentido un análisis de las mismas se puede realizar restando una de otra, suponiendo que existe un $100 \%$ de transformación de CdO en CdS y un crecimiento adicional de pequeñas partículas de CdS, según la expresión (15):

$$
\mathrm{I}(\text { matriz }+\mathrm{CdO})-\mathrm{K} \mathbf{I}(\text { matriz }+\mathrm{CdS})=\mathbf{I}(0) \exp \left(-\mathrm{R}_{\mathrm{gq}}^{2} \mathbf{q}^{2} / 3\right)
$$

Eligiendo un factor $\mathrm{K}$ de forma que se obtenga el mejor comportamiento de Guinier posible, siendo $\mathrm{R}_{\mathrm{g}}$ el radio de giro y q el módulo del vector de difusión:

$$
\left[\mathrm{q}=\frac{4 \pi \operatorname{sen} \theta / 2}{\lambda}\right]
$$

siendo $\theta$ el ángulo de difusión y $\lambda$ la longitud de onda de los

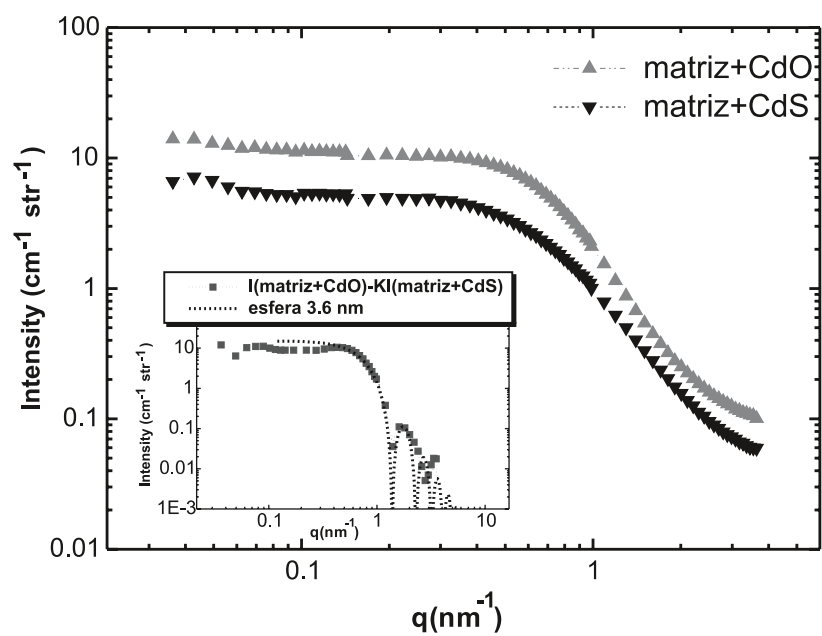

Figura 1. Intensidades experimentales en escala log-log de las dos muestras estudiadas. La figura interior representa el resultado del análisis de la ecuación [1] en trazo de puntos, junto con la intensidad difundida por una esfera del mismo tamaño, $3.6 \mathrm{~nm}$, en trazo continuo.

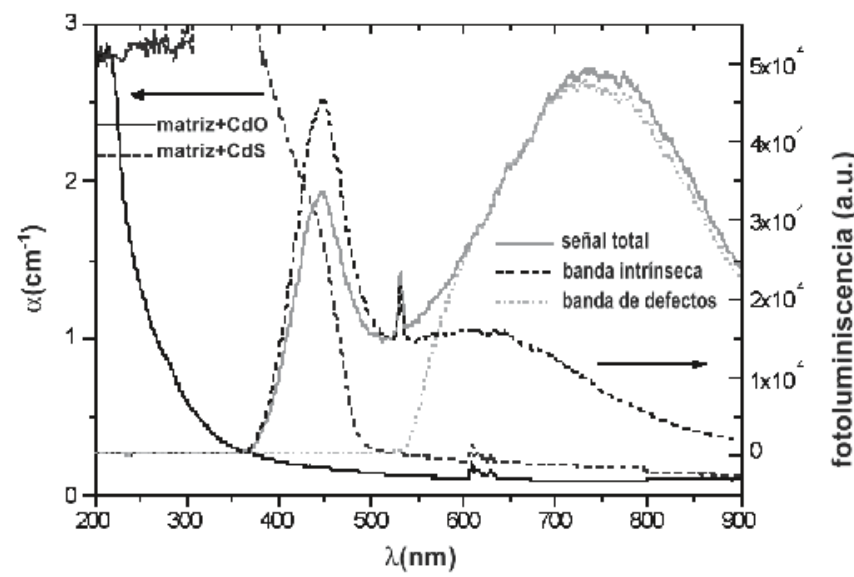

Figura 2. Coeficiente de absorción, escala izquierda y señal de fotoluminiscencia, escala derecha, para las muestras estudiadas.

neutrones. Los resultados del análisis de la ecuación (1) se muestran en la figura interior a la Fig.1, donde se aprecia la amplia región de Guinier en la zona de bajo valores de q y los máximos característicos de la dispersión debida a una esfera (discontinuidad en la función de Bessel para $\mathbf{q R}+\operatorname{tg}(\mathbf{q R})$ en la zona de altos valores de $q$, lo que destaca el carácter seudoesférico de los nanocristales. Por otro lado, la envolvente de dichos máximos permite estimar una pendiente de -4 , lo que nos informa de un buen comportamiento de la ley de Porod debido a superficies nítidas de los cristales, no presentando fluctuaciones de densidad de la longitud de difusión en la frontera entre los cristales y la matriz dieléctrica. Una vez cuantificados los valores a partir de la expresión [1], se obtiene un radio de giro de $2.8 \mathrm{~nm}$ que le corresponde una esfera de $3.6 \mathrm{~nm}$ de radio (ver interior a la Fig.1) donde se ilustra la difusión de una esfera de $3.6 \mathrm{~nm}$.

El comportamiento desde el punto de vista óptico se muestra en la Fig.2 que representa los espectros de absorción óptica de ambas muestras junto con la señal de fotoluminiscencia de la matriz+CdS sólo. Comparativamente hemos incluido el 
espectro de la matriz+CdO para indicar la ausencia de absorción en el visible. Se han incluido también las señales de fotoluminiscencia correspondientes a las bandas intrínseca y de defectos mediante filtración de la radiación, Schott OG-550 y BG-14, respectivamente.

El espectro de absorción CdS+matriz pone de manifiesto que los nanocristales son los responsables del hombro de absorción en torno a $2.76 \mathrm{eV}$. Este desplazamiento hacia el azul en 230 meV respecto de la banda del CdS masivo en $2.53 \mathrm{eV}$, está inducido por el tamaño nanométrico de los cristales, $\mathbf{R}(\mathrm{CdS})=3.6$ $\mathrm{nm} \approx \mathrm{a}_{\mathrm{B}}(\mathrm{Bohr})=3.2 \mathrm{~nm}$, confirmando el confinamiento cuántico de los estados fotoexcitados creados, 1EHP y 2EHP enlazados, responsables del comportamiento óptico no-lineal en estos materiales. La señal de fotoluminiscencia presenta dos bandas de emisión, una debida a transiciones intrínsecas con un marcado pico centrado en $2.76 \mathrm{eV}$, que no tiene procesos de migración en la red cristalina, emitiendo fotones sin pérdida de energía en el proceso de recombinación. Sin embargo, no ocurre así para la señal debida a los defectos superficiales, cuya banda se produce a más baja energía, $1.65 \mathrm{eV}$, con una anchura de unos $620 \mathrm{meV}$. El desplazamiento hacia el rojo que muestra esta banda es debido a transiciones internas interbandas de los portadores antes de recombinarse.

El estudio del comportamiento óptico no-lineal se realizó a partir del crecimiento de la señal de fotoluminiscencia en función de la excitación óptica. Dicho estudio se realizó mediante el método de rendija de longitud variable. Para valores pequeños de la rendija, si el crecimiento de la señal es superlineal indica la existencia de ganancia óptica neta de los procesos de recombinación radiativos. La Fig.3 presenta el registro del crecimiento de la fotoluminiscencia en función del tamaño de la rendija, desde $50 \mu \mathrm{m}$ hasta $2 \mathrm{~mm}$ de abajo hacia arriba, la anchura de la rendija es de $20 \mu \mathrm{m}$. La señal corresponde a la banda intrínseca filtrada ópticamente la banda de defectos con un filtro Schott BG-14. Se observa un desplazamiento hacia el rojo del máximo, centrado en $450 \mathrm{~nm}$, debido a la saturación del número de portadores en los diferentes niveles de energía accesibles. La señal de fotoluminiscencia se relaciona con el coeficiente de ganancia a partir de la expresión ${ }^{14}$ :

$$
\mathbf{I}_{\mathrm{AL}}=\frac{\mathbf{I}_{\mathrm{SP}}}{\mathrm{gL}}\left(\mathrm{e}^{\mathrm{gL}}-\mathbf{1}\right)
$$

donde $\mathrm{I}_{\mathrm{AL}}$ y $\mathrm{I}_{\mathrm{SP}}$ son la intensidad amplificada y espontánea, respectivamente. L la apertura de la rendija y g el coeficiente neto de ganancia que incluye las pérdidas debidas a procesos no-radiativos. Utilizando el algoritmo de LevenbergMarquardt (16) se ajusta la ecuación [3] a las intensidades experimentales para cada longitud de onda dando el coeficiente de ganancia y la intensidad espontánea.

El espectro de ganancia de dicho ajuste se muestra en el interior a la Fig.3, para las primeras 8, 10 y 12 tamaños de la rendija, la variación es consecuencia de la saturación, que en estos sistemas se produce en torno a gL $>2$ (17), esto es, para rendijas mayores de $350 \mu \mathrm{m}$. Como puede verse el espectro de la ganancia muestra las mismas características que la señal de fotoluminiscencia en el mismo rango espectral, siendo poco intensa para bajas energías que corresponde al resto de la banda de defectos filtrada. El pico correspondiente a la parte de altas energías, con una anchura de $300 \mathrm{meV}$, es atribuible tanto a transiciones de 1EHP al nivel fundamental de energía como de 2EHP, siendo más probable ésta última, con la consiguiente formación de 1EHP más un fotón. Esta amplificación dará lugar a una absorción inducida de la muestra que se

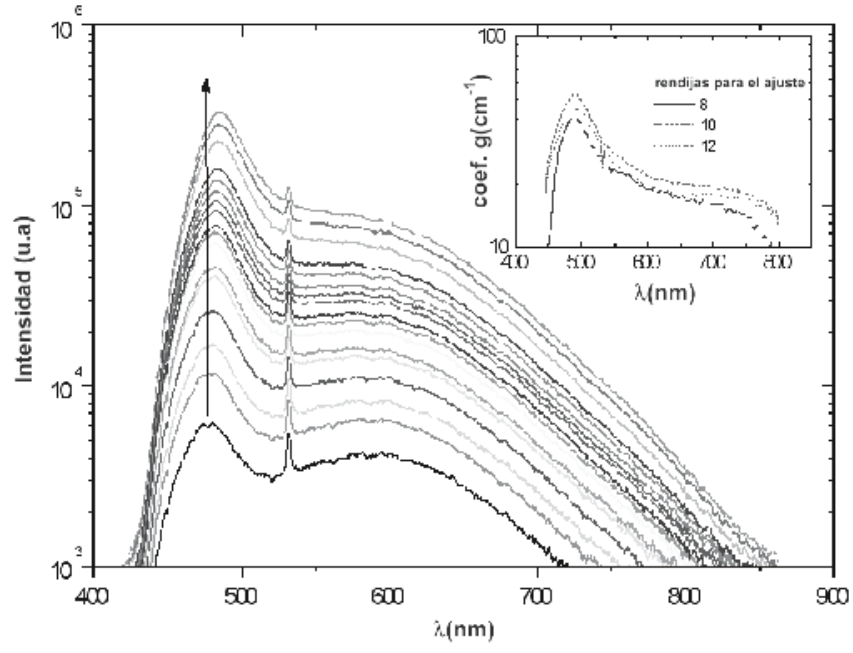

Figura 3. Crecimiento de la señal de fotoluminiscencia en función de la anchura de la rendija L, de abajo hacia arriba. La figura interior muestra el espectro de ganancia óptica según el número de rendijas utilizadas en el ajuste de la ecuación [3].

asigna mayoritariamente a la generación de biexcitones en el nanocristal de CdS, semiconductor de dimensión cero, como consecuencia existirá una variación no-lineal en el índice de refracción debido a la ionización de los portadores fotoexcitados, produciendo los fenómenos de autofocalización y autodesfocalización. Por tanto, la existencia de amplificación óptica, g>0, imprescindible para producir luz láser, es indicativo de un comportamiento óptico no-lineal debido a los nanocristales de $\mathrm{CdS}$ en la matriz dieléctrica, debido principalmente a la formación de biexcitones.

\section{CONCLUSIONES.}

Con el presente estudio podemos concluir que mediante la técnica sol-gel es posible obtener nanocristales de CdS inmersos en una matriz porosa de sílice. Mediante técnicas de SANS hemos podido aislar la señal de los nanocristales, que presentan una distribución homogénea y un tamaño de $3.6 \mathrm{~nm}$ cuasiesféricos. Las medidas realizadas de la densidad óptica y de la señal de fotoluminiscencia, permiten concluir un alto grado de confinamiento cuántico de los estados fotoexcitados en los nanocristales de CdS, que actúan como semiconductores de dimensión cero, quantum dots, embebidos en la matriz dieléctrica de sílice. Mediante la técnica de rendija de longitud variable se ha podido obtener el espectro de ganancia óptica en un amplio rango espectral con un valor máximo de $\mathrm{g}=60 \mathrm{~cm}^{-1}$, limitado éste por los fenómenos de saturación y los defectos superficiales en el nanocristal. La amplificación óptica demuestra el comportamiento óptico no-lineal de los nanocristales de CdS como semiconductores de dimensión cero y por tanto prometedores materiales de uso en dispositivos fotónicos.

\section{AGRADECIMIENTOS.}

Este trabajo ha sido realizado en el marco del proyecto de la CICyT (MAT98-0798) y las medidas en Berlín bajo el programa TMR/LSF (ERBFMGECT950060) de la Comisión Europea. 


\section{BIBLIOGRAFÏA}

1. U. Woggon in: Optical Properties of Semiconductors Quantum Dots, Springer Tracts in Modern Physics (vol. 136). (Springer, Berlin, 1997).

2. N. Peyghambarian, S. W. Koch and A. Mysyrowicz in: Introduction to Semiconductors Optics. (Prentice-Hall, New Jersey,1993).

3. J.I. Pankove in: Optical Processes in Semiconductors. (Dover, New York, 1971).

4. N.F. Borrelli, D.W. Hall, H.J. Holland and D.W. Smith, "Quantum confinement effects of semiconducting microcrystallites in glass", J. Appl. Phys. 61, 5399-5409 (1987).

5. L. Efros and A. Efros, "Interband absorptin of light in a semiconductor sphere", Sov. Phys. Semicond. 16, $772-775$ (1982).

6. N. Peyghambarian, S.W. Koch, B. McGinnis, K. Kang, Sandalphon, Y.Z. Hu, S.H. Risbud, L-Ch. Liu, A. Mysyrowicz and D. Hulin, "Hole-State mixing and nonlinear optical properties of semiconductor quantum dots", Sci. and Tech. of Mesoscopic Struc., S. Namba, C. Hamaguchi, T. Ando (Eds.) (Springer-Verlag, Tokyo, 1992) pp. 443-449.

7. Y.A. Vlasov, K Luterova, I. Pelant, B. Hönerlage and V.N. Astratov, "Enhancement of optical gain of semiconductors embedded in three-dimensional photonic crystals", Appl. Phys. Lett. 71, 1616-1618 (1997).

8. M. Sheik-Bahae, A.A. Said and E.W. Van Stryland, "High sensivity single beam $n_{2}$ measurement", Opt. Lett. 14, 955-957 (1989).

9. N. de la Rosa-Fox, A. Santos, M. Piñero, L. Esquivias, L. Gago-Duport and
E. Hoinkis, "SANS study of CdS and CdSe quantum dots crystal growth in a silica matrix by sol-gel", J. Sol-Gel Sci. Tech. 13, 629-633 (1998).

10. E. Blanco, L. Esquivias, R. Litran, M. Piñero, M. Ramirez-del-Solar and N. de la Rosa-Fox, "Sonogels and Derived Materials". Appl. Organometal. Chem. 13, 399-418 (1999).

11. R. Erce-Montilla, M. Piñero, A. Santos, N. de la Rosa-Fox and L. Esquivias, "Control growth of PbS nanocrystals in silica sono-ormosil", J. Mat. Res. (to be published).

12. R. Litran, R. Alcántara, E. Blanco, and M. Ramirez-del-Solar, “Confinement of CdS nanocrystals in a sonogel matrix", J. Sol-Gel Sci. Tech. 8, 275-283 (1997).

13. G. Banfi, V. Degiorgio and B. Speit, "Neutron scattering investigation of the structure of semiconductor-doped glasses", J. Appl. Phys. 74, 6925-6935 (1993).

14. K.L. Shaklee, R.E. Nahory and R.F.Leheny, “Optical gain in semiconductors", J. of Lumin. 7, 284-309 (1973).

15. A. Craievich, N. de la Rosa-Fox, E. Blanco, M. Piñero, M. Ramirez-del-Solar and L. Esquivias, "CdS nanocrystals embedded in silica sonogel", NanoStruct. Mat. 5, 363-372 (1995).

16. D. Marquardt, “An algorithm for least-squares estimation of nonlinear parameters", SIAM J. on Appl. Math, 11, 431-441 (1963).

17. J. Butty, N. Peyghambarian, Y.H. Kao and J.D. Mackenzie, "Room temperature optical gain in sol-gel derived CdS quantum dots", Appl. Phys. Lett. 69, 3224-3226 (1996) 